\title{
SOUND FIELD MEASUREMENTS BASED ON RECONSTRUCTION FROM LASER PROJECTIONS
}

\author{
Yasuhiro Oikawa, Makoto Goto, Yusuke Ikeda, Toshikazu Takizawa and Yoshio Yamasaki \\ Global Information and Telecommunication Studies, Waseda University, Tokyo, Japan \\ \{oikawa, goto, ikeda, toshijazz, yamasaki\}@acoust.rise.waseda.ac.jp
}

\begin{abstract}
In this paper, we describe some new sound field measurement methods by using laser Doppler vibrometer (LDV). By Irradiating the reflection wall with a laser, we can observe the light velocity change that is caused by the refractive index change from the change in air density. It means that it is possible to observe the change of the sound pressure.

We measured a sound field projection on a 2-D plane using a scanning laser Doppler vibrometer (SVM) which can visualize a sound field. And we made a 3-D sound field reconstruction from some 2-D laser projections based on the computed tomography (CT) techniques. We made the reconstruction image for the sound field near the loudspeaker or in the room.
\end{abstract}

\section{INTRODUCTION}

It is effective to visualize the sound field information for understanding the behavior of the sound field. Many researchers have recently studied on numerical computation and visualization of sound fields because it has become easier to use the high performance computers [1]. It has been also researched to visualize sound fields by the real-world experiences. It is difficult to visualize the real-world sound fields because we have to measure the sound pressure or intensity correctly at so many points using microphones. On the other hand, we suggested the four point microphone method, which can earn the spatial information from four impulse responses which are measured using nearly located four microphones [2]. We have measured many sound fields by using this method.

We usually use a number of microphones to measure sound fields. But there is another choice as to measure sound pressure level at broad sound field using laser Doppler vibrometer (LDV) instead of microphones. It is possible to measure the average sound pressure level over the laser path. The measured value includes all information on the path of laser. If we have any measured data on some area from different direction using LDV, we can estimate sound field in the area without measuring at many points using microphones. This kind of signal processing is known as computed tomography (CT) based on the reconstruction from projections. For example, the $\mathrm{X}$-ray CT is widely used in the medical field to observe inside the body without contract and destruction. We observe the sound field by using laser CT technique instead of X-ray CT.

In this paper we describe the sound pressure measurement using LDV and also suggest the sound field reconstruction from laser projection. We are able to visualize 2-D or 3-D sound fields in the real world.

\section{SOUND PRESSURE MEASUREMENT USING LASER DOPPLER VIBROMETER}

It is possible to observe the sound pressure from the change of the refractive index of air [3]. From the state equation for the adiabatic change of the gas, we get the relation between the volume change and the refractive index change as

$$
\frac{\Delta V}{V}=-\frac{\Delta n}{n-1},
$$

where $V$ is the volume and $n$ is the refractive index.

$$
\begin{aligned}
& \frac{P}{P_{0}}=-\gamma \frac{\Delta V}{V} \text { and } \\
& P_{0} \gamma=c^{2} \rho
\end{aligned}
$$

where $P_{0}$ is the atmosphere pressure, $\gamma$ is the specific heat ratio, $\rho$ is the density, and $c$ is the sound velocity. From Eq.(1), (2), and (3),

$$
\Delta n=\frac{n-1}{c^{2} \rho} P \text {. }
$$

If we pass the laser from the LDV to the sound field against the reflection wall and measure the reflected laser, we can observe, by using LDV, the refractive index change which produces the sound pressure changes according to distance. So the relationship of the refractive index change and the distance change is 


$$
\Delta n \cdot l=n \cdot \Delta l .
$$

Using LDV the refractive index change is

$$
\Delta n=\frac{n}{2 \pi f l} v_{L D V},
$$

where $l$ is the distance of laser path, $f$ is the frequency of sound and $v_{L D V}$ is the measured velocity. From Eq.(4) and (6), we get

$$
P=\frac{n}{n-1} \frac{c^{2} \rho}{2 \pi f l} v_{L D V} .
$$

\section{RECONSTRUCTION FROM LASER PROJECTIONS}

The reconstruction from projections was suggested by Radon [4], and this kind of signal processing is known as CT based on the reconstruction from projections [5]. If we enter the pencil beam X-ray into the material,

$$
I=I_{0} \exp \left(-\int_{-\infty}^{\infty} f(s) d s\right),
$$

where $I$ is the observed intensity, $I_{0}$ is the irradiation intensity, and $f(s)$ is the absorption index. From Eq.(8),

$$
p=-\log _{e} \frac{I}{I_{0}}=\int_{-\infty}^{\infty} f(s) d s .
$$

If we take a projection from a point $(r, \theta)$ on the rotating coordinate system,

$$
p(r, \theta)=\int_{-\infty}^{\infty} f(x, y, \theta) d s,
$$

where $s=x \cos \theta+y \sin \theta$.

To get $f(x, y)$, we use the convolution back projection method (CBP method). This method includes two processes. The first process is the convolution calculation and the second process is the back projection calculation. On the convolution process,

$$
q(r, \theta)=p(r, \theta) * g(r),
$$

where $q(r, \theta)$ is the rectilinear projection and $g(r)$ is the rectilinear function. On the back projection process,

$$
f(x, y)=\int_{0}^{\pi} q(r, \theta) d \theta .
$$

This means that the sum of $q(r, \theta)$ for all $\theta$ is the reconstruction. We usually measure the projection and calculate the back projection discretely. We have the discrete projection data $p(k a, j \Delta \theta)$, where $k=0, \pm 1, \cdots, \pm m, j=0,1, \cdots, n-1, a$ and $\Delta \theta$ are the sampling distance for $r$ and $\theta$, respectively. The rectilinear function

$$
g(k a)=\frac{2}{\pi^{2} a^{2}\left(1-4 k^{2}\right)} .
$$

is used well [5].

In this paper we use $v_{L D V}$, which is the measured values by $L D V$, instead of $p$.

\section{EXPERIMENTS}

We did some experiments in real world based on the mentioned method. First we measured the sound pressure using a scanning laser Doppler vibrometer (SVM) and visualized the sound field. Secondarily, we reconstructed the sound fields using $\mathrm{CT}$ technique. To consider the relations between the number of projections and resolutions, we reconstructed the sound field from projections of different number. Then we reconstructed the sound field near the loudspeaker from laser projections based on CT technique. Finally we reconstructed the room sound field.

\subsection{Visualization of sound field using SVM}

We visualized the sound field by sound pressure measurement method, which is mentioned in the session 2. We measured the sound pressure at many points using SVM and visualized a sound pressure distribution. Figure 1 shows the experiment condition. We put a loudspeaker between the reflection wall and the laser head. In these experiments we used the ordinary loudspeaker, which is MSP-10 from YAMAHA, and the flat panel loudspeaker, which is W-3232 from WASEDA EE [6]. The measurement signal is $4 \mathrm{kHz}$ sinusoidal tone. We used the concrete heavy wall of the reverberant chamber as the reflection wall. It was confirmed that this wall did not move using another LDV.

Figure 2 and Fig. 3 show the sound field made by the ordinary loudspeaker and the flat panel loudspeaker, respectively.

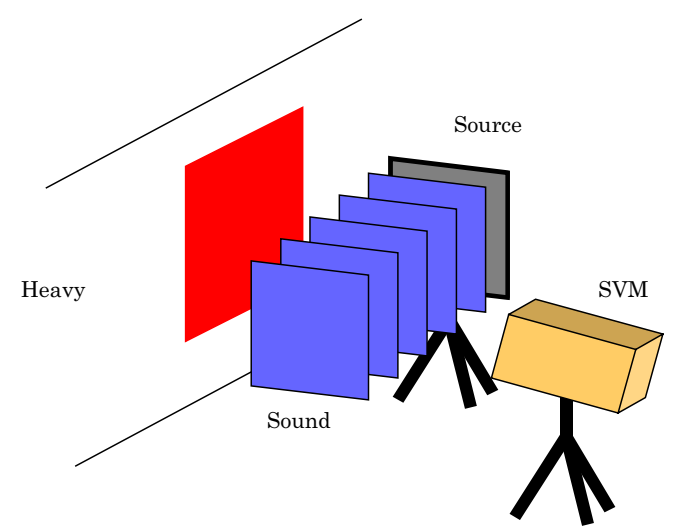

Fig.1. Measurement conditions. The loudspeaker is located between SVM and the reflection wall. 


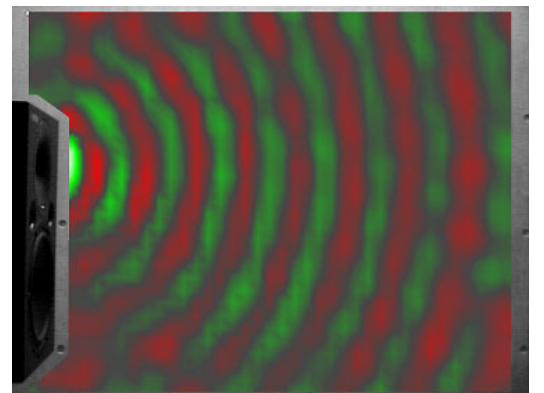

Fig.2. Sound field made by the ordinary loudspeaker. The spherical sound wave is constructed.

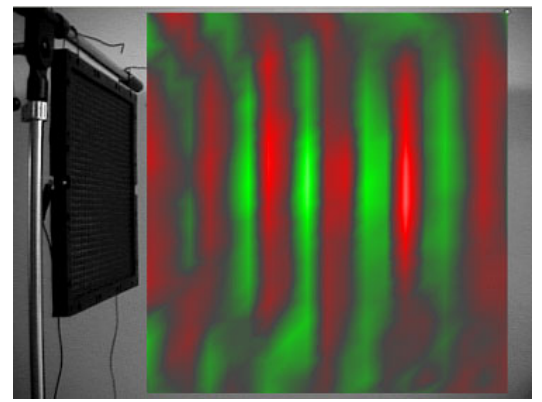

Fig.3. Sound field made by the flat panel loudspeaker. The planed sound wave is constructed.

\subsection{Resolution of sound field reconstruction}

We made the reconstruction of the sound field by the method mentioned in session 3 and considered the reconstruction resolution. We assumed that the laser projections from every directions were the same and calculated the sound field reconstruction.

Figure.4, 5, and 6 show the reconstruction from 360, 36, and 18 projections, respectively. The reconstruction from 36 projections is almost the same as that of 360 projections inside the target area.

\subsection{Sound field reconstruction of near distance field}

We underwent the sound field reconstruction experiments based on the laser CT technique. We also used the ordinary loudspeaker (YAMAHA MSP-10) (condition A) and the flat panel loudspeaker (WASEDA EE W-3232) (condition B). The loudspeaker was located on the table toward the ceiling in the echoic chamber shown in Fig.7. Because the symmetric condition was satisfied for these speakers, we rotated them instead of LDV head. Laser was scanned $7 \mathrm{~cm}$ high over the loudspeaker. Figure 8 shows the measurement condition. The number of scanning points was 110 and 100 on the condition $A$ and $\mathrm{B}$, respectively. The distance between each measured points was almost $10 \mathrm{~mm}$. In these experiments we took 36 laser projections and reconstructed from them. The measurement signal was $4 \mathrm{kHz}$ sinusoidal.
Figure 9 and 10 show the 3-D sound field reconstruction on the condition $\mathrm{A}$ and $\mathrm{B}$, respectively. The shape of the vibrating part of loudspeaker is put on the $x-y$ plane of each Figure. We irradiated the reflection wall with a laser of fan-beam shape, but we assumed that the laser beam goes on parallel way.

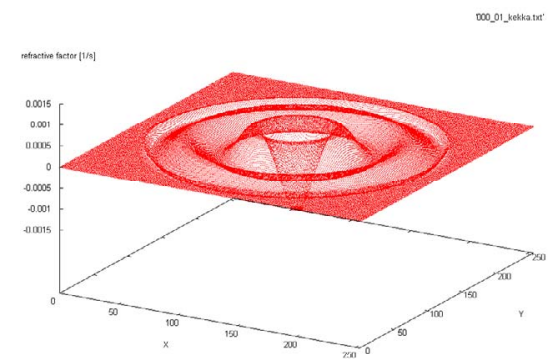

Fig.4. Reconstruction from 360 projections.

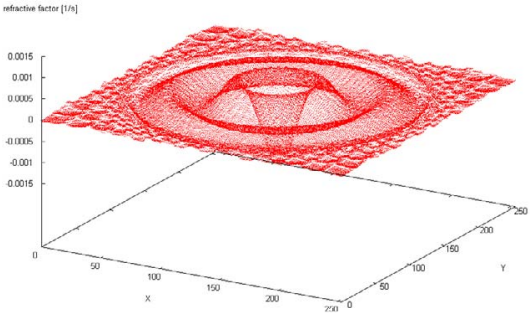

Fig.5. Reconstruction from 36 projections.

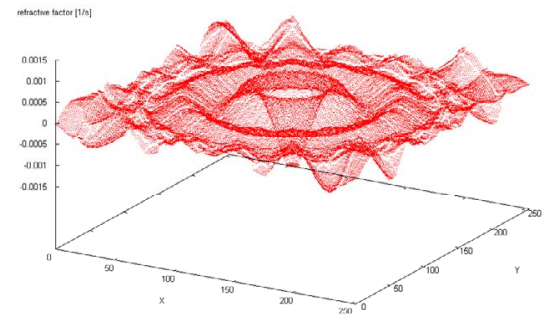

Fig.6. Reconstruction from 18 projections.

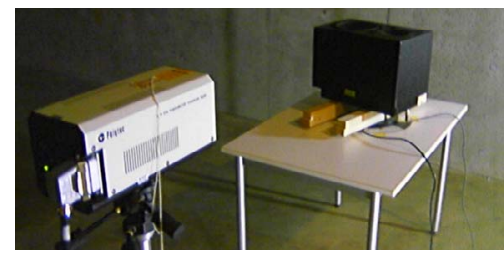

Fig.7. Picture of experiment. The loudspeaker is located on the table toward the ceiling.

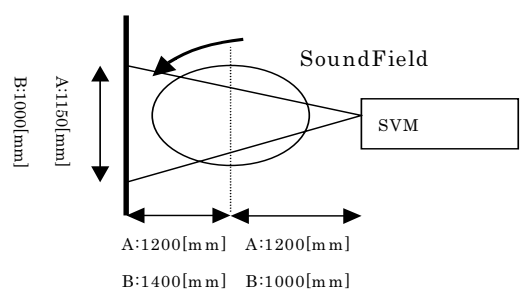

Fig.8. Measurement Conditions. 


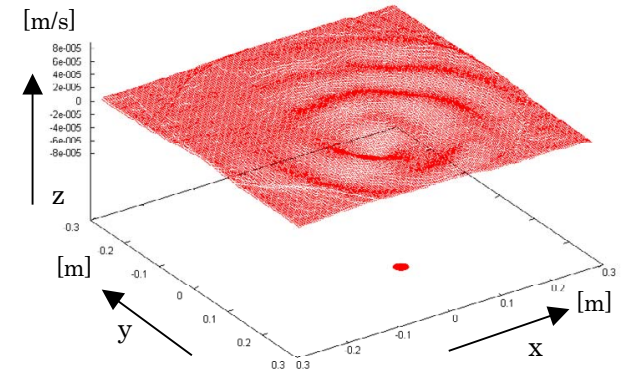

Fig.9. Reconstruction from 36 projections. The spherical sound wave is made by the ordinary loudspeaker.

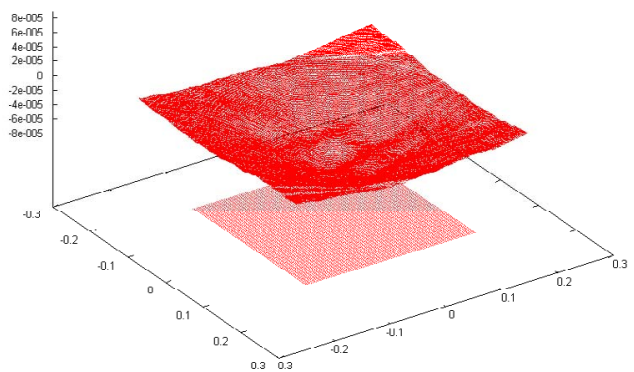

Fig.10. Reconstruction from 36 projections. The flat sound wave is made by the flat panel loudspeaker.

\subsection{Sound field reconstruction in the room}

We reconstructed the sound field in the room from laser projections. We took this measurement in the echoic chamber with the sound absorbing materials. We used the non-directional loudspeaker as the point sound source. We also took 24 laser projections around the loudspeaker. Figure 11 shows the sound field reconstruction. We could find the direct sound near the loudspeaker and the reflected sounds in the outside.

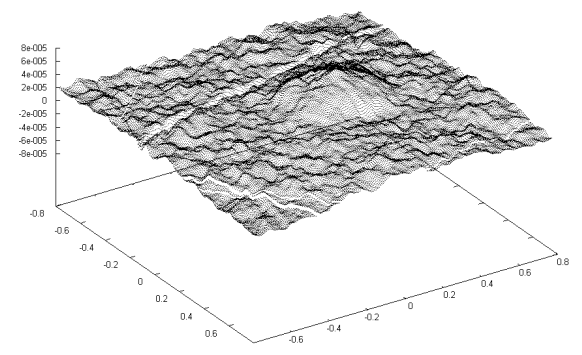

\section{CONCLUSIONS}

In this paper, we have described some methods for sound field measurement using SVM. We could measure the sound pressures using LDV and visualize the sound pressure distribution. We also were able to calculate the reconstruction of sound field from the laser projections using CT technique. This time, we assumed that the laser beam goes on parallel way. The cone-beam algorithm for $\mathrm{CT}$ is suggested in [7]. We expect that the additional improvement can be achieved in real-world measurement. We are currently working on this.

\section{REFERENCES}

[1] T. Yokota, M. Iiduka, S. Sakamoto, H. Tachibana, "Differences of Sound Pressure Level Distribution Due to the Variations of Room Shape," in Proc. WESPAC VIII, 2003.

[2] Y. Yamasaki and T. Itow, "Measurement of spatial information in sound fields by closely located four point microphone method,” J. Acoust. Soc. Japan (E), Vol.10, No.2, pp.101-110, 1989.

[3] K. Nakamura, "Measurements of high power ultrasound in the air through the modulation in the refractive index of air," Tech. report IEICE, US2001-9, pp.15-20, 2001.

[4] J. Radon, "Uber die Bestimmug von Funktionen durch Ihre integralwerte Laengs Geweisser Mannigfaltigkeiten," Berichte Saechsishe Acad. Wissenschaft.Math.Phys., Klass 69, pp.262277, 1917.

[5] L. A. Shepp and B. F. Logan, "The Fourier reconstruction of a head section," IEEE Trans. Nucl. Sci., NS-21, pp. 21-43, 1974. [6] Y. Yamasaki, H. Nakajima, M. Okazaki, and Y. Kato, "Transducers considering energy saving -super conductive and electrostatic loudspeaker driven by 1-bit amplifier-," in Proc. Int. Cong. Acoust., Vol. IV, pp.2427-2432, 2004.

[7]L. A. Feldkamp, L. C. Davis, and J. W. Kress, "Practical cone-beam algorithm," J. Opt. Soc. Am., A/Vol.1, No.6, pp.612619, 1984.

\section{APPENDIX}

We attached some movies in DVD-ROM proceedings. Table.1 shows movie file names in DVD-ROM on the comparison of flat panel loudspeaker with ordinary loudspeaker. Table. 2 also shows movie file names on the comparison of same phase driving with inverse phase driving using two loudspeakers. The file name "ct_room_divx.avi" in DVD-ROM shows the movie reconstructing the sound field inside the room.

Fig.11. Reconstruction of the room sound field.

Table.1. Movie files on the comparison of flat panel loudspeaker with ordinary loudspeaker.

\begin{tabular}{|c|c|c|c|}
\hline Sp. & Visualization & $\mathrm{CT}(10 \mathrm{~cm}$ distance from $\mathrm{Sp})$. & $\mathrm{CT}(50 \mathrm{~cm}$ distance from Sp.) \\
\hline Flat Panel & flat4k_divx.avi & ct_flat $4 \mathrm{k} 10 \mathrm{~cm}$ _divx.avi & ct_flat $4 \mathrm{k} 50 \mathrm{~cm}$ _divx.avi \\
\hline NS10M & ns $10 \mathrm{~m} 4 \mathrm{k}_{-}$divx.avi & ct_ns $10 \mathrm{~m} 4 \mathrm{k} 10 \mathrm{~cm}$ _divx.avi & ct_ns $10 \mathrm{~m} 4 \mathrm{k} 50 \mathrm{~cm}$ _divx.avi \\
\hline $\mathrm{Sp}$ & Visualization & $\mathrm{CT}(10 \mathrm{~cm}$ distance from $\mathrm{Sp})$. & $\mathrm{CT}(50 \mathrm{~cm}$ distance from $\mathrm{Sp})$. \\
\hline Same Phase & stereo_same_4k_divx.avi & ct_stereo_same $4 \mathrm{k} 10 \mathrm{~cm}$ divx.avi & ct_stereo_same $4 \mathrm{k} 50 \mathrm{~cm}$ divx.avi \\
\hline Inverse Phase & stereo_inv_4k_divx.avi & ct_stereo_inv4k10cm_divx.avi & ct_stereo_inv4k50cm_divx.avi \\
\hline
\end{tabular}

